\title{
High Quality Cross Sections of Low Melting Point Samples Prepared with Cryo Ion Slicer - Broad Ar Ion Beam Milling Apparatus with a Newly Developed Specimen Cooling Unit
}

\author{
Y.Nakajima*, M.Shibata*, H.Matsushima*, T.Suzuki*, and Natasha Erdman** \\ * SEM Application Group, JEOL Ltd.Musashino3-1-2, Akishima, Tokyo, 196-8558 JAPAN \\ **JEOL USA Inc., 11 Dearborn Rd. Peabody, MA. 01960
}

Ion Slicer (IS) is an instrument for the preparation of TEM lamellas and SEM cross-sections by employing an Ar broad ion beam [1]. It has been getting quite popular in many fields because of its ease of use and high quality results. However, it is difficult to mill low melting point materials by the Ar broad ion beam because of thermal damage during the milling. Therefore, we have developed a specimen cooling unit for IS that maintains specimen at low temperature during the ion milling to avoid the thermal damage. The Cryo Ion Slicer (CIS) is a modified IS, and has a specimen cooling system as an additional function.

Figure 1 shows the external appearance of CIS. Figure 2 shows the principle of the basic unit of IS. The specimen for the IS has to be pre-thinned to the size of $2.8 \mathrm{~mm}$ (long) x $1.0 \mathrm{~mm}$ (wide) $\times 0.1 \mathrm{~mm}$ (thick) before Ar ion beam milling by the IS. The accelerating voltage of the Ar ion beam can be chosen between 1 and $8 \mathrm{kV}$. The Ar ion beam is automatically and continuously rocked with an angle up to $\pm 6^{\circ}$ during the specimen milling. The Ar broad ion beam mills both sides of the specimen not covered by the shielding belt. As the milling proceeds, a thin section is made under the shielding belt. Without rocking the Ar ion beam, a thin specimen with the same thickness as the shielding belt can be obtained. Figure 3 shows the specimen cooling system of the CIS. The specimen can be cooled down to $-120^{\circ} \mathrm{C}$ (approximately $1 \mathrm{hr}$ to reach the temperature). Before taking out the specimen after the ion milling, the cooling conductors should be disconnected from the specimen stage and the specimen should be warmed up to the room temperature by the heater as shown in Figure 3-B (approximately 30 min to warm up the specimen).

We have previously reported a successful result of CIS for solder specimen [2]. The melting point of solder is $183^{\circ} \mathrm{C}$. This time we tried to make a cross-section of wood metal. The wood metal is an alloy of $\mathrm{Bi} 50 \%, \mathrm{~Pb} 26.7 \%$, $\mathrm{Sn} 13.3 \%$ and $\mathrm{Cd} 10 \%$, and is a typical low melting point material. Its melting point is $70^{\circ} \mathrm{C}$, and is much lower than that of the solder. Figures $4 \mathrm{~A}$, 4B and 4C are the SEM backscattered electron (BSE) images of cross-sections of wood metal. From the elemental analysis with EDS, various phases present in the material are marked. The BSE image shown in Figure 4A is the ion milling result with the CIS with an accelerating voltage $4 \mathrm{kV}$. The BSE image shown in Figure $4 \mathrm{~B}$ is the result of the conventional Cross-Section Polisher (CP) at $4 \mathrm{kV}$ [3]. Comparing these two images, it is clearly noticed that the cross-section made by the conventional CP has many voids (arrows) on the surface due to the temperature increase. On the other hand, the BSE image shown in Figure 4C shows a cross-sectional plane of wood metal which was made by an ultramicrotome. There are no voids on the surface because of no temperature increase. However, the crystalline structure in bismuth-rich phase looks different from the result of the CIS (allows). It is likely due to the deformation of crystals caused by the knife pressure of the ultramicrotome.

We will also report successful results of CIS polishing on a variety of additional materials. 
Reference:

1) A. Yasuhara, JEOL News, vol. 40 number 1, 46-49 (2005)

2) H. Matsushima, et al., IMC17, I18, 13 (2010)

3) M. Shibata, JEOL News, vol. 39 (1), 28-31 (2004)

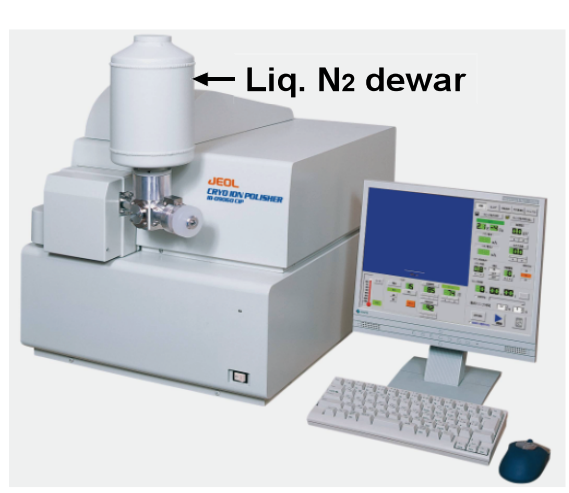

Fig. 1 Cryo Ion Slicer

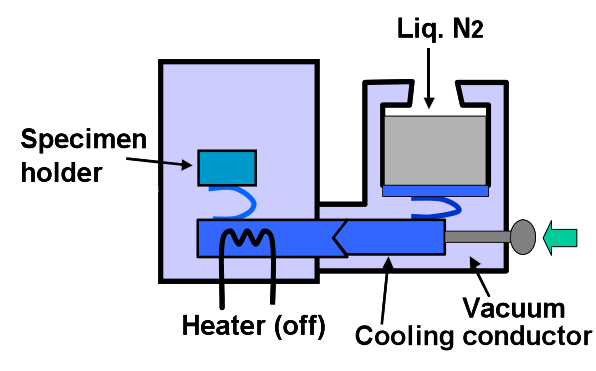

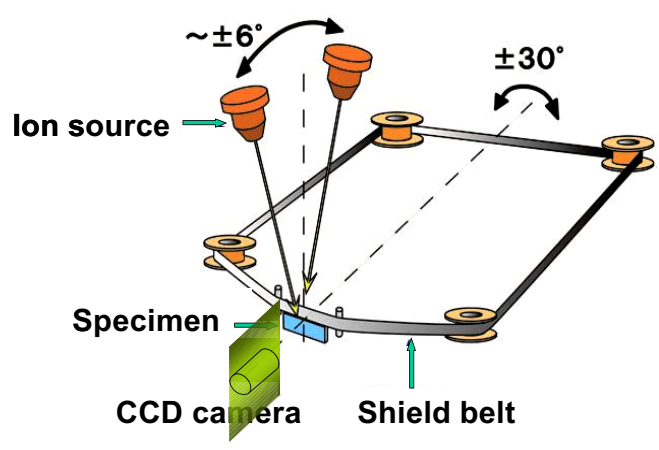

Fig. 2 Principle of Ion Slicer

Fig. 3 Principle of specimen cooling

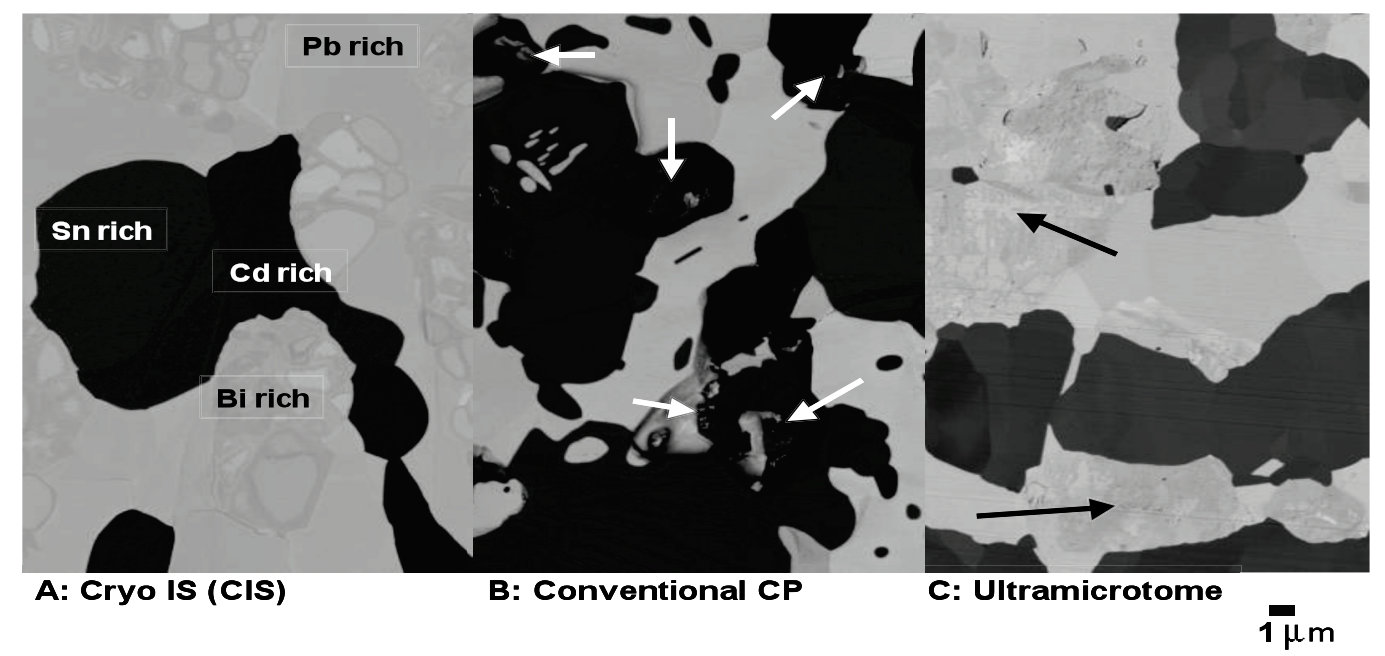

Fig. 4 Comparison of various sample preparation methods. 